\title{
XP Enigma - Um Jogo Educacional Não Digital para Apoio ao Ensino de Métodos Ágeis: Uma Análise Temporal da Motivação e Aprendizagem
}

\author{
Pablo Schoeffel \\ Departamento de Engenharia de Software \\ UDESC/Universidade do Estado de Santa Catarina \\ Ibirama - Santa Catarina - Brasil \\ pablo.schoeffel@udesc.br
}

\section{RESUMO}

Este artigo descreve um jogo educacional não digital, baseado em uma atividade de caça ao tesouro, para apoiar o ensino de metodologias ágeis, mais especificamente de práticas e valores do Extreme Programming (XP). 0 trabalho descreve o detalhamento do jogo, uma avaliação pós-teste realizada com 20 estudantes utilizando o modelo de avaliação para jogos educacionais MEEGA, assim como uma avaliação da percepção de 46 egressos sobre o jogo dois a quatro anos após a participação. Como resultados, foi identificado um alto índice de satisfação em termos de motivação e experiência do usuário, um aumento significativo de conhecimento em todos os conteúdos avaliados. Além disso, foi identificada a percepção positiva dos egressos quanto à motivação e aprendizagem propiciados pela atividade, mesmo alguns anos após a participação desses estudantes no jogo.

\section{PALAVRAS-CHAVE}

Jogo Sério, Métodos Ágeis, Gerenciamento de Projetos, XP, Extreme Programming.

\section{Introdução}

O constante aumento na utilização de metodologias ágeis (MA) de desenvolvimento de software, sendo inclusive uma das causas para a melhoria dos índices de sucesso de projetos de software [1], demonstra a importância na disseminação dessas metodologias em currículos de cursos de graduação e pósgraduação.

Segundo o State of Agile Report [2], 95\% das empresas utilizam ou utilizaram práticas ágeis em alguma equipe e as metodologias ágeis mais utilizadas, individualmente ou em

Fica permitido ao(s) autor(es) ou a terceiros a reprodução ou distribuição, em parte ou no todo, do material extraído dessa obra, de forma verbatim, adaptada ou remixada, bem como a criação ou produção a partir do conteúdo dessa obra, para fins não comerciais, desde que sejam atribuídos os devidos créditos à criação original, sob os termos da licença CC BY-NC 4.0. EduComp'21, Abril 27-30, 2021, Jataí, Goiás, Brasil (On-line)

(C)2021 Copyright mantido pelo(s) autor(es). Direitos de publicação licenciados à Sociedade Brasileira de Computação (SBC). conjunto com outras, são o SCRUM (76\%), Kanban (17\%) e XP (9\%).

Porém, embora sejam utilizadas por muitas empresas [3], as metodologias ágeis ainda são recentes, comparando-se com outras abordagens e uma das causas de fracassos em implementação de MA são a falta de conhecimento e experiência [3]. Dessa forma, é de suma importância que o ensino dessas metodologias seja atrativo e eficiente. Nesse contexto, surge a importância de o professor repassar os assuntos utilizando um conjunto variado de estratégias de ensino. No âmbito dos cursos de computação, métodos ágeis são geralmente ensinados em disciplinas de Engenharia de Software, Gerenciamento de Projetos ou, mais raramente, em disciplinas específicas.

Segundo Ibrahim [4], a disciplina de Gerenciamento de Projetos é teórica, em sua natureza, sendo um desafio ensinar seu conteúdo, uma vez que pode ser desinteressante quando ensinado teoricamente.

Da mesma forma, iniciativas de ensino e aprendizagem em Engenharia de Software (ES) requerem novas e não tradicionais formas de pensar [5]. Bavota, Lucia e Fasano [6] relatam ainda que "um dos principais desafios no ensino de ES dentro de um curso de graduação é proporcionar aos alunos experiências significativas, os quais irão achar úteis quando entram no mercado de trabalho".

Atividades práticas permitem que o aluno seja mais proativo e se envolva com as atividades ao mesmo tempo em que aprende. A combinação de várias abordagens pode ser considerada uma chave para facilitar o processo de ensino e aprendizagem [4].

Segundo Goldman et al. [7], apesar de MA estar se mostrando eficiente no desenvolvimento de software, a maioria dos cursos universitários ainda são baseadas em métodos antigos, de estilo peso pesado.

A mudança para uma abordagem ágil na indústria cria a necessidade de uma mudança similar na educação em Engenharia de Software [8]. Ao mesmo tempo, muitos trabalhos [9] [10] têm mostrado a eficiência de jogos educacionais no ensino de Engenharia de Software.

Portanto, nota-se a necessidade do ensino de MA nos cursos de graduação e pós-graduação em computação, porém percebem-se os grandes desafios e a necessidade de utilizar diferentes abordagens, com ênfase para abordagens práticas. Algumas abordagens têm sido produzidas, a fim de criar e 
avaliar jogos ou atividades vivenciais para o ensino de Métodos Ágeis [11] [12] [13]. Porém, muitos dos trabalhos utilizam a estratégia de simulação, principalmente utilizando os processos e práticas utilizadas no SCRUM. Poucos trabalhos abordam as práticas de engenharia ou outras metodologias, como o XP.

Beck [14] descreve o XP como uma disciplina de desenvolvimento de software que organiza as pessoas para produzir código de alta qualidade de forma mais produtiva. 0 $\mathrm{XP}$ tenta reduzir o custo das mudanças em requisitos, tendo vários pequenos ciclos de desenvolvimento. O XP possui cinco valores norteadores: comunicação (colaborativa e oral), simplicidade (minimizar a quantidade de trabalho não realizado), feedback (constante feedback em diversas dimensões: cliente, testes, time), coragem (esforço para encorajar a equipe a refatorar, dar feedback, etc.) e respeito (respeitar o time e a si próprio).

Além dos valores, o XP possui doze práticas principais: o cliente sempre disponível, uso de metáforas no projeto, jogo do planejamento, entregas pequenas e rápidas, testes de aceitação, programação em pares, refatoração, integração contínua, propriedade coletiva, design simples, ritmo sustentável e padrões de código.

Para suprir essa necessidade, esse trabalho apresenta um jogo baseado em uma atividade de caça ao tesouro, que visa consolidar a aprendizagem de diversas práticas das metodologias ágeis, com foco no XP e nas práticas pouco abordadas por outros trabalhos.

Esse trabalho está estruturado da seguinte forma: na Seção 2 são apresentados os trabalhos relacionados; na Seção 3 é explicado o jogo XP Enigma; na Seção 4 são apresentados os métodos de aplicação e avaliação do jogo; na Seção 5 são apresentados os resultados; e na Seção 6 são descritas as considerações e conclusões.

\section{Trabalhos Correlacionados}

Apesar do aumento da utilização de métodos ágeis pela indústria e da crescente adoção de jogos sérios como estratégias de ensino, ainda percebe-se carências de jogos para o ensino de métodos ágeis. Existem diversos jogos para ensino de gerenciamento de projetos em geral, muitos baseado em simulações [15] . Dos trabalhos encontrados para o ensino de métodos ágeis, a maioria utiliza o framework SCRUM [16] [11] [17] [18] [10] [12] [19], relacionados ou simulando atividades como: estimativa e priorização de histórias, reunião de planejamento, sprints e retrospectiva.

Diversos trabalhos realizaram revisões sistemáticas sobre jogos sérios ou educacionais em Engenharia de Software [15] [20] [21] . Dessas revisões, Rodrigues et al. [20] identificaram 29 trabalhos relacionados a Gerenciamento de Projetos e Souza et al. [21] identificaram 92 trabalhos (59\% do total de trabalhos encontrados no estudo) relacionados a processos de software, que envolve o gerenciamento de projetos. Portanto, o uso de jogos para ensino de GP tem mostrado ser amplamente utilizado. Porém, especificamente para ensino de metodologias ágeis, Souza et al. [21] encontraram somente sete trabalhos.

Porém, algumas práticas e valores ágeis, principalmente da metodologia XP, estão pouco presentes nesses trabalhos.
Supõe-se que o fato de serem práticas abstratas e difíceis de serem demonstradas com situações reais de desenvolvimento de software no ambiente educacional, dificultam o processo de ensino-aprendizagem.

Dentre os trabalhos encontrados, Bergin e Grossman [22] descrevem o jogo Extreme Construction, que é uma simulação de um projeto para construção de artefatos, como pontes, castelos, escolas, etc., fazendo uso dos princípios da metodologia ágil XP. Outro jogo relacionado é o XPGame [13]. Esse jogo faz referência à metodologia Extreme Programming, porém limita-se ao processo de estimativa, jogo do planejamento, sprints, testes de aceitação e validação, similar ao processo do SCRUM.

Przybyłek e Zakrzewski [23] propuseram e avaliaram um framework composto de um conjunto de oito jogos colaborativos para apoiar a aprendizagem de engenharia de requisitos ágeis. A análise da auto avaliação de oito participantes mostraram resultados positivos quanto à promoção da participação dos clientes e comprometimento dos participantes. Porém, além de limitar-se a requisitos ágeis, não foram avaliadas a percepção dos estudantes e a evolução da aprendizagem.

Outros trabalhos, como descritos em Parsons [24], enfocam em uma única prática, como programação em pares, reuniões diárias em pé (standup meetings), estratégia do time e refatoração. Por exemplo, o jogo Collaborative-Origami que contempla a programação em pares, porém não foram encontrados trabalhos descrevendo sua aplicação e validação. Também não foram encontrados outros jogos que abordassem as seguintes práticas do XP: metáfora, ritmo sustentável e padrão de código.

A Tabela 1 mostra um comparativo dos dois trabalhos correlatos mais similares ao proposto, com relação à cobertura das práticas do XP.

Tabela 1: Cobertura das práticas XP nos trabalhos correlatos

\begin{tabular}{|l|l|l|l|}
\hline \multicolumn{1}{|c|}{ Prática } & \multicolumn{1}{|c|}{$\begin{array}{c}\text { XP } \\
\text { Game }\end{array}$} & $\begin{array}{c}\text { Extreme } \\
\text { Construction }\end{array}$ & $\begin{array}{c}\text { Xp } \\
\text { Enigma }\end{array}$ \\
\hline Jogo do Planejamento & Sim & Sim & Sim \\
\hline Entregas rápidas & Sim & Sim & Sim \\
\hline Design Simples & - & Sim & Sim \\
\hline Testes de Aceitação & Sim & Sim & Sim \\
\hline Refatoração em & - & Sim & Sim \\
\hline $\begin{array}{l}\text { Programação } \\
\text { pares }\end{array}$ & - & Sim & Sim \\
\hline Propriedade coletiva & - & Sim & Sim \\
\hline Integração contínua & - & Sim & Sim \\
\hline Cliente disponível & Sim & Sim & Sim \\
\hline Ritmo sustentável & - & - & Sim \\
\hline Metáforas & - & - & Sim \\
\hline Padrão de Código & - & - & - \\
\hline
\end{tabular}

Nesse sentido, o jogo XP Enigma apresentado nesse trabalho tem como principais diferenciais abordar, em um 
único jogo educacional, a maioria das práticas do XP, incluindo aquelas que não são contempladas por jogos atualmente disponíveis e que são, em geral, difíceis de serem implementadas em atividades práticas em sala de aula.

\section{XP Enigma}

O XP Enigma é um jogo não digital, colaborativo e disputado entre equipes, que promove a busca a uma mandala ${ }^{1}$ perdida, baseada numa série de atividades e enigmas que as equipes precisam decifrar e realizar em equipe. Optou-se pela criação de um jogo não digital por vantagens já relatadas por [15], como a menor complexidade no desenvolvimento, maior flexibilidade para ajustes e adaptações, além de promover e encorajar habilidades de comunicação, interações e habilidades sociais, que são condizentes com os objetivos de aprendizagem almejados.

\subsection{Objetivos de Aprendizagem}

O jogo tem como público alvo estudantes da disciplina de Métodos Ágeis, Engenharia de Software ou Gerenciamento de Projetos de cursos de graduação e pós-graduação. Como objetivos de aprendizagem principais, tem-se: i) executar atividades utilizando práticas, princípios e valores ágeis (Extreme Programming e SCRUM), através de um projeto fictício e de atividades lúdicas; ii) comparar práticas ágeis com práticas "tradicionais"; iii) entender os benefícios das práticas ágeis; iv) fazer analogia das atividades realizadas sob o ponto de vista do processo de desenvolvimento de software.

\subsection{Contexto}

O jogo é uma espécie de caça ao tesouro, cujo objetivo das equipes participantes é desvendar todas as charadas/enigmas (no formato de histórias de usuário), a fim de descobrir antes das demais equipes uma mandala perdida. 0 jogo é executado em três ou mais iterações, que serão executadas por todos os estudantes. Sugere-se que a turma seja dividida em grupos de cinco a dez estudantes, tendo, em cada grupo, os papéis relacionados na Tabela 2 .

\section{Tabela 2: Papéis do XP Enigma}

\begin{tabular}{|l|l|}
\hline Papel & Atividade \\
\hline 1 Líder & $\begin{array}{l}\text { Alocar atividades na iteração 1. Nas demais } \\
\text { iterações: coordenar reuniões de } \\
\text { planejamento e retrospectiva, ajudar a } \\
\text { resolver impedimentos. NÃO executa } \\
\text { tarefas }\end{array}$ \\
\hline 1 Cliente & $\begin{array}{l}\text { Compartilhado entre as equipes, valida as } \\
\text { entregas/verifica resultado das integrações }\end{array}$ \\
\hline $\begin{array}{l}\text { Integradores } \\
\text { ou 2 }\end{array}$ & $\begin{array}{l}\text { Realiza as atividades de integração (junção } \\
\text { de duas ou mais atividades) e fornece } \\
\text { feedback para time }\end{array}$ \\
\hline
\end{tabular}

1 Espécie de medalha com um diagrama composto de formas geométricas concêntricas

\begin{tabular}{|l|l|l|}
\hline Pares (time) & $\begin{array}{l}\text { Execução das tarefas, planejamento e } \\
\text { retrospectiva }\end{array}$
\end{tabular}

0 papel de cliente pode ser executado pelo próprio professor da disciplina e os papéis de integrador e os pares (membros dos times) podem ser em número diferenciado, de acordo com quantidade de alunos da turma. 0 importante é possuir pelo menos dois grupos com cinco ou mais alunos e que cada grupo defina um local (Quartel General - QG) para realizar suas atividades.

O objetivo final dos grupos será encontrar e entregar para o cliente uma mandala perdida. A atividade inicia com a explicação do professor que, em seguida, solicita para as equipes definirem os papéis. A partir daí, o líder recebe o escopo do projeto, que consiste em um conjunto de dicas e charadas que, ao final, o levarão ao tesouro perdido (mandala).

\subsection{Estrutura e Fluxo do Jogo}

A atividade é realizada em fluxos/iterações com tempo prédefinido de, aproximadamente, vinte minutos de execução. A fim de evidenciar alguns diferenciais das práticas e valores ágeis, algumas regras são pré-definidas, visando diferenciar atividades realizadas com práticas tradicionais e com práticas ágeis em diferentes iterações:

1. Na Iteração 1, o time só pode se comunicar de forma escrita;

2. Na Iteração 1 , a integração só pode ser feita após a execução da iteração;

3. Na Iteração 1, cada membro irá realizar as tarefas sem apoio dos demais;

4. Na Iteração 1, serão atribuídas pessoas às tarefas no início, pelo Líder;

5. Na Iteração 1, o contato com o cliente será somente na fase de revisão (após a execução);

6. Caso todos os membros da equipe não estejam no QG ao final do tempo estabelecido, para cada minuto de atraso até que todos cheguem, a equipe perderá 2 minutos do próximo ciclo/iteração.

As dicas e charadas são o mecanismo principal do jogo, a partir das quais os times avançam em busca do objetivo final. A fim de prover dinamismo à atividade e também para relacionar com práticas e valores ágeis, as dicas/charadas são classificadas quanto ao tipo e podem também ter um objetivo de aprendizagem específico. Quanto ao tipo, são divididas em:

- $\quad$ Artes: requer uso de conhecimentos ou habilidades artísticas, como desenho, montagem, colagem, etc. (ex: Você precisará se localizar no campus/sala para algumas atividades. Faça uma planta do campus numa folha de papel);

- Lógica, matemática e geometria: requer raciocínio lógico, cálculos ou análises espaciais (ex: próximo número de uma determinada sequência numérica); 
- Conhecimentos gerais: relacionadas a conhecimentos gerais, como: esporte, política, idiomas, cultura, natureza, etc. (ex: Nome dado a mulher com cabelo ruivo (popular) + Nome da filha adolescente da família que protagoniza o seriado "Os Simpsons");

- Integração: envolvem a integração de outras duas ou mais dicas/charadas (ex: local representado pelas dicas 1 , 2 e 3);

- Enigma: envolvem uso de metáforas e conhecimento popular (ex: Não penso nem reflito, mas conhecimento eu transmito, como se fosse uma lua irradiando sua claridade numa noite escura).

Quanto aos objetivos de aprendizagem, as dicas/charadas podem ser classificadas em:

- Refatoração: requer que alguma atividade anteriormente realizada seja melhorada ou reestruturada;

- Design simples: envolve o desenvolvimento de alguma atividade cujo resultado será mais efetivo se realizado de forma simples;

- Metáfora: envolve o uso de metáforas para descrever algo, como enigmas;

- Integração contínua: tarefas de integração que, caso realizadas de forma contínua, geram resultados melhores.

O Apêndice I mostra as dicas/charadas utilizadas, as quais podem e devem ser adaptadas para a realidade de cada contexto, devido às restrições do espaço físico disponível. No exemplo mostrado, estão indicadas, em negrito, as respectivas respostas, as quais os estudantes devem acertar. É importante que as dicas e charadas sejam de conhecimento comum e com tipos variados, para evitar que alguma das equipes tenha uma vantagem maior por ter algum conhecimento específico.

A partir das dicas, os grupos devem descobrir uma chave para acessar a última dica, que indica a localização da mandala, com ajuda de outras dicas.

A fim de inserir práticas ágeis, a partir da iteração 2 , as equipes devem trabalhar em pares (pode-se, por exemplo, solicitar que eles amarrem os punhos em duplas), as tarefas de integração podem ser realizadas a qualquer momento, assim como a comunicação e validação com o cliente. A Tabela 3 mostra as fases e tempo planejado para cada fase. 0 objetivo de cada iteração é representar um sprint, contendo o planejamento, execução, revisão e retrospectiva.

\section{Tabela 3: Fases do XP Enigma}

\begin{tabular}{|l|r|}
\hline Fase & Tempo \\
\hline $0-$ Explicação Inicial & $10 \mathrm{~min}$. \\
\hline 1.1 - Planejamento Inicial & $5 \mathrm{~min}$. \\
\hline 1.2 - Execução da Iteração 1 & $10 \mathrm{~min}$. \\
\hline 1.3 - Integração da Iteração 1 & $2 \mathrm{~min}$. \\
\hline 1.4 - Revisão e retrospectiva da Iteração 1 & $5 \mathrm{~min}$. \\
\hline 2.1 - Planejamento Iteração 2 & $2 \mathrm{~min}$. \\
\hline 2.2 - Execução da Iteração 2 & $10 \mathrm{~min}$. \\
\hline
\end{tabular}

\begin{tabular}{|l|r|}
\hline 2.3 - Revisão e Retrospectiva da Iteração 2 & $5 \mathrm{~min}$. \\
\hline 3.1 - Planejamento Iteração 3 & $2 \mathrm{~min}$. \\
\hline 3.2 - Execução da Iteração 3 & $10 \mathrm{~min}$. \\
\hline 3.3 - Revisão e Retrospectiva da Iteração 3 & $5 \mathrm{~min}$. \\
\hline 4 - Retrospectiva da atividade (debriefing) & $10 \mathrm{~min}$. \\
\hline
\end{tabular}

A atividade possui duração de, aproximadamente, oitenta minutos, podendo ser aplicada para uma ou duas interações a mais, caso necessário. A Figura 1 mostra os estudantes em dois momentos: no QG do grupo trabalhando para resolver as atividades e alcançando o objetivo final de encontrar a mandala, escondida numa lixeira no estacionamento do campus.

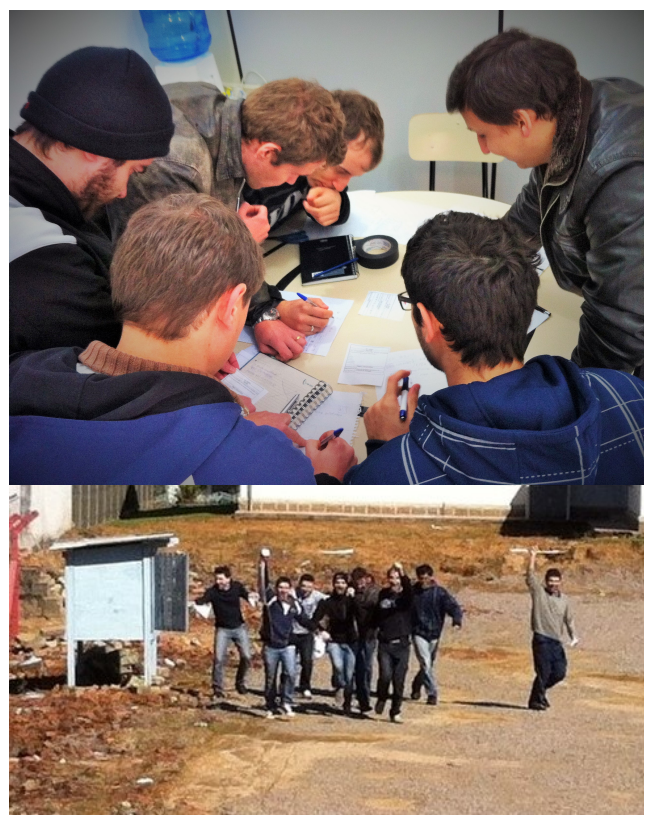

Figura 1: Alunos realizando as atividades do jogo no QG (a) e encontrando a mandala (b)

Na fase 0 , o professor explica funcionamento da dinâmica e divide os alunos em times. As fases 1.1 a 1.4 refere-se à primeira iteração, na qual são impostas algumas restrições (diferentes ou contrárias ao desenvolvimento ágil). Na fase 1.1, cada time deve escolher os papéis, incluindo o líder que, nessa fase, é o único a ler as estórias. Em seguida, o líder deve atribuir tarefas ao time, para serem realizadas no primeiro ciclo. Nessa fase, os cartões de tarefas não são abertos ainda, podendo ser abertos somente na execução (fase 1.2). Na fase 1.2, cada membro do time deve finalizar a sua tarefa para fazer próxima. A comunicação só pode ser escrita e os resultados devem ser escritos atrás da estória, quando necessário. As tarefas concluídas devem ser entregues ao integrador, ao final do ciclo. $\mathrm{Na}$ fase 1.3, o integrador realiza as tarefas de integração, que dependem da execução de outras tarefas. Os membros do time poderão refatorar somente suas próprias tarefas, se necessário. Na fase 1.4, o líder faz a validação com o cliente. Em casos de não adequação, a tarefa deve ser refeita/complementada no próximo ciclo. 0 time dá um feedback com relação ao primeiro 
ciclo e discutem possíveis melhorias, porém toda comunicação deve ser somente escrita.

As fases 2.1 a 2.3 referem-se à segunda iteração, onde são incentivadas práticas mais alinhadas aos métodos ágeis. $\mathrm{Na}$ fase 2.1, as tarefas podem ser abertas sobre mesa e todo o time faz o planejamento de prioridades e se auto organiza para escolha das atividades. A comunicação pode ser realizada de forma oral (face a face). $\mathrm{Na}$ fase 2.2, as atividades são executadas em pares (uma por vez). Um par pode pedir apoio para outros membros do time e os resultados devem ser escritos atrás da estória, quando necessário. As tarefas concluídas podem ser entregues ao integrador e integradas continuamente. Quaisquer dúvidas podem ser validadas com o cliente durante o ciclo. Os membros do time podem refatorar qualquer tarefa a qualquer tempo, se necessário. Na fase 2.3 , o time faz validação com o cliente. Em casos de não adequação, a tarefa deve ser refeita/complementada no próximo ciclo por qualquer membro do time. 0 time se reúne para discutir pontos positivos e negativos, em relação à segunda iteração.

As fases 3.1 a 3.3 referem-se à terceira iteração e seguem as mesmas regras da segunda iteração (2.1 a 2.3). Caso seja necessário, a atividade pode ter a quarta e quinta iteração, seguindo as mesmas regras da segunda, até a conclusão do projeto.

$\mathrm{Na}$ fase 4, o professor deve discutir a prática com os estudantes, obtendo um feedback dos participantes e fazendo analogias das práticas e processo realizados com atividades de Engenharia de Software. A retrospectiva visa avaliar, juntamente com os participantes, as atividades realizadas no jogo, propondo uma reflexão a fim de que os objetivos de aprendizagem sejam atendidos. São exemplos de questões a serem discutidas com os estudantes:

- Quais as maiores dificuldades encontradas?

- Quais práticas ágeis vocês identificaram na atividade?

- Quais diferenças vocês notaram entre a primeira iteração e as demais?

- Que comparação podemos fazer da atividade realizada com o desenvolvimento de software?

Para nortear a discussão da atividade com os estudantes, é importante mapear quais práticas ágeis e em que situação, fazem parte do jogo. A seguir, são descritas as boas práticas sugeridas na metodologia XP com as respectivas práticas ágeis presentes no XP Enigma.

1. O cliente sempre disponível: o jogo permite a interação com cliente, para sanar dúvidas e validar partes das atividades. Para enfatizar a importância dessa prática, na primeira iteração essa prática não é permitida;

2. Uso de metáforas no projeto: algumas das tarefas foram elaboradas utilizando metáforas, por exemplo: a charada que informa que a mandala está localizada no campus assim como o Ushuaia está para a América do Sul. O objetivo foi mostrar como uma metáfora pode ser usada para descrever um requisito;

3. Jogos de planejamento: a cada iteração, a equipe tem um tempo para priorizar, replanejar as atividades e verificar dúvidas com o cliente;
4. Pequenas versões: a divisão da atividade em iterações, simulando sprints, faz com que as equipes entreguem partes pequenas a cada vez, compostas das atividades finalizadas;

5. Testes de aceitação: todas as tarefas finalizadas são apresentadas para o cliente, o qual informa se a atividade é válida e está aprovada;

6. Integração contínua: algumas atividades, durante o processo, necessitam da integração entre outras atividades. É enfatizada a importância da integração contínua, restringindo as tarefas de integração para serem feitas somente após a primeira iteração e nas demais interações sendo realizadas durante a execução;

7. Design simples: algumas atividades (ex: atividade que solicita um desenho do campus da universidade) devem ser executadas prevendo a simplicidade, sob pena da equipe perder tempo sem necessidade;

8. Refatoração: algumas atividades precisam ser melhoradas em iterações posteriores. Por exemplo, o desenho da planta do campus, a fim de conseguir se localizar e posicionar os pontos cardeais e numeração das salas, para identificar mais rapidamente a localização;

9. Programação em pares: todas as atividades, a partir da segunda iteração, são realizadas em pares;

10. Propriedade coletiva: todas as atividades podem ser realizadas por todos os membros. Isso foi evidenciado, restringindo na primeira iteração que os membros trocassem atividades ou se ajudassem;

11. Ritmo sustentável: essa prática está relacionada com a restrição de todos os membros do time estarem no QG ao final de cada integração, sob pena de perder tempo na próxima iteração.

A única das doze boas práticas originais do XP não abordada diretamente no jogo é "padrão de código". Além dessas práticas, alguns dos princípios ágeis que não estão contemplados nas práticas originais do XP, também estão presentes no XP Enigma e podem ser utilizados para discussão com estudantes:

1. Criar projetos em torno de indivíduos motivados, onde as decisões são tomadas por toda equipe. Embora tenha o papel do líder, a partir da iteração 2 as decisões são tomadas em equipe;

2. Comunicação face-a-face é a mais eficiente: é explorada a diferença de comunicação ao restringir, na iteração 1, a comunicação escrita e permitir, a partir da iteração 2 , a comunicação face a face;

3. Equipes auto organizadas: também a partir da iteração 2 é permitido que o time possa escolher como trabalhar nas atividades, ao contrário da iteração 1, onde um líder atribui as tarefas para cada membro;

4. Reflexão em intervalos regulares: para cada iteração, é incluída uma fase de retrospectiva, onde o time reflete sobre os pontos positivos e negativos e define estratégias de melhoria para a próxima iteração.

Com isso, percebe-se que muitas das doze práticas e dos princípios sugeridos pela metodologia XP são contemplados no jogo, além de alguns dos princípios do manifesto ágil, os quais devem ser discutidos ao final da atividade, para que os alunos 
relacionem os conceitos à prática executada e percebam seus benefícios.

\section{Metodologia}

Esse trabalho relata o desenvolvimento e avaliação de um jogo educacional para ensino de métodos ágeis. 0 jogo é utilizado como ferramenta complementar a outras abordagens, a fim de aplicar alguns conceitos e, dessa forma, ajudar os alunos a reforçarem o conhecimento e o entendimento do assunto. Com isso, o jogo faz parte de uma unidade instrucional de uma disciplina de Métodos Ágeis, Gerenciamento de Projetos ou Engenharia de Software.

O XP Enigma foi aplicado em três turmas da disciplina de Métodos Ágeis, com carga horária de 30 horas/aula, do curso de especialização lato sensu em Engenharia de Software. A disciplina abordou os valores e princípios das metodologias ágeis, além da demonstração de algumas das metodologias: SCRUM, XP, Modelagem Ágil, Lean. O conteúdo foi abordado utilizando aulas tradicionais (exposição pelo professor), além de outras dinâmicas e práticas. Ao final, no último dia da disciplina, foi aplicado o jogo XP Enigma, com objetivos de fixar e aplicar alguns dos conceitos repassados.

Nas duas primeiras turmas, foram realizadas aplicações piloto, sendo realizadas melhorias de acordo com o feedback dos estudantes e avaliação do instrutor. Nas aplicações piloto foram ajustados, principalmente, os tempos de cada fase e iteração, a complexidade e a descrição das atividades/charadas. Na terceira turma, foi aplicado um questionário pós-teste para os 20 alunos participantes, conforme modelo MEEGA de avaliação de jogos educacionais proposto por [25], avaliando os seguintes aspectos: motivação (satisfação, confiança, relevância e atenção), experiência do usuário (imersão, interação, desafio, diversão e competência) e aprendizagem (com base na taxonomia de Bloom). 0 questionário consiste de 27 itens divididos nos três componentes descritos, com opções de resposta seguindo uma escala Likert com alternativas de discordo fortemente $(-2)$ até concordo fortemente $(+2)$.

A fim de avaliar a efetividade do jogo, após um período de tempo, foram enviados questionários a todos os 62 alunos egressos ( 2 a 4 anos após a participação na disciplina) que participaram do jogo. Para isso, foi desenvolvido um questionário online, respondido por 46 estudantes, composto pelos seguintes itens:

- Métodos de ensino mais lembrados (questão aberta);

- $\quad$ Aprendizagem promovida pelo jogo XP Enigma;

- $\quad$ Motivação promovida pelo jogo XP Enigma;

A consistência interna do questionário foi avaliada pelo cálculo do Coeficiente Alpha de Cronbach, cujo resultado foi = 0.8831, mostrando-se confiável, segundo o intervalo de valores aceitáveis do alpha (0.70 a 0.95$)$ descrito por [26].

\section{Resultados e Discussão}

O jogo foi aplicado em três turmas, totalizando 62 estudantes. As duas primeiras turmas foram utilizadas como aplicação piloto, sendo realizadas melhorias e mudanças de acordo com o feedback dos estudantes e avaliação do professor. Na terceira turma, foi realizada uma avaliação pós-teste com 20 estudantes, seguindo o modelo MEEGA para avaliação de jogos educacionais.

\subsection{Avaliação Pós-teste}

A atividade foi realizada dividindo uma turma com vinte alunos em duas equipes de dez alunos, onde para cada equipe foram escolhidos o líder e o integrador. Como resultado, além do ambiente de integração e descontração, os alunos conseguiram encontrar a mandala perdida em quatro interações.

Considerando os valores da escala Likert $(-2 \mathrm{a}+2)$, a média da avaliação foi de 1,83, o que representa uma aceitação de cerca de $96 \%$. A Figura 2 mostra os aspectos relacionados à motivação com a atividade e a Figura 3 mostra os resultados dos aspectos relacionados à experiência do usuário.

Percebe-se, conforme Figura 2, que o único item que teve avaliação negativa (dois estudantes) foi "É por causa do meu esforço pessoal que consigo avançar no jogo". Da mesma forma, o único item sobre experiência do usuário com avaliação negativa foi "Consegui atingir os objetivos do jogo por meio das minhas habilidades" (Figura 3). Supõe-se que essa percepção é devido ao fato do avanço no jogo depender fortemente do trabalho em equipe.

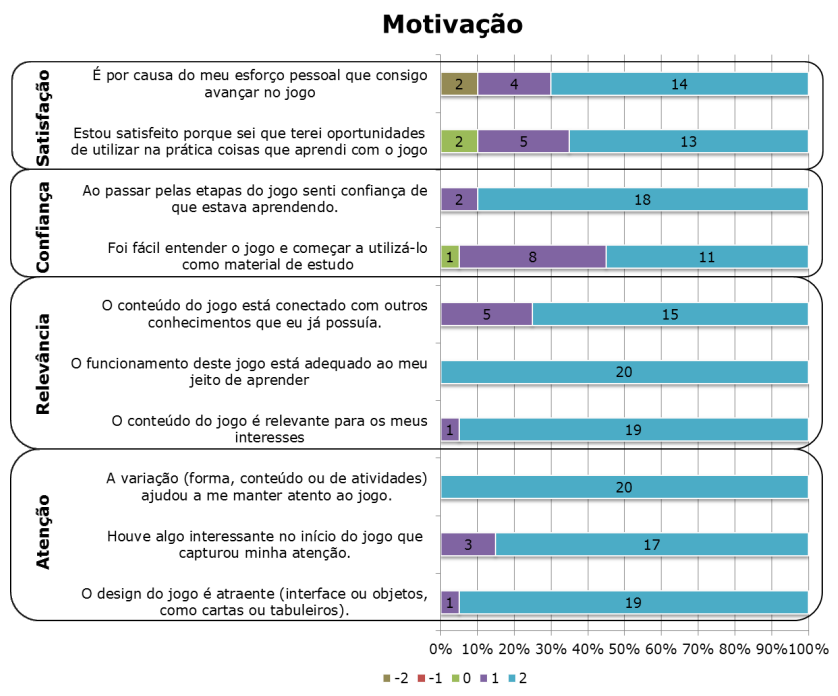

Figura 2: Avaliação da motivação do XP Enigma 


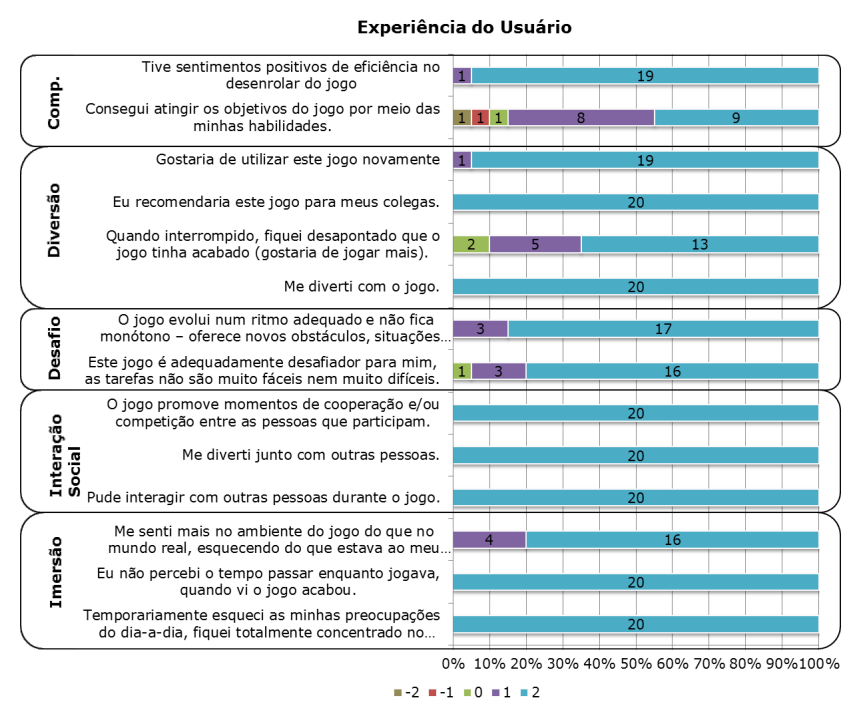

Figura 3: Avaliação da experiência do usuário

Com relação ao terceiro aspecto, aprendizagem, a Figura 4 mostra a avaliação geral da percepção de aprendizagem. Podese perceber que todas as avaliações foram positivas, sendo $85 \%$ de avaliações extremamente positivas (+2).

Para aqueles conteúdos que são foco principal do XP Enigma e que raramente foram encontrados nos trabalhos correlatos, foram coletados os dados de percepção de evolução da aprendizagem (antes e após a participação no XP Enigma). Na Tabela 5, é exibida a média de cada um dos conteúdos avaliados, mostrando um significativo nível de concordância com a contribuição da atividade para a evolução da aprendizagem em todos os conteúdos avaliados.

Foi identificado que, para todos os conteúdos e níveis cognitivos, houve uma diferença estatisticamente significante $(\mathrm{p}<0.01)$ entre o conhecimento antes e depois da participação no jogo, aplicando o teste $t$-student para amostras pareadas (Tabela 5).

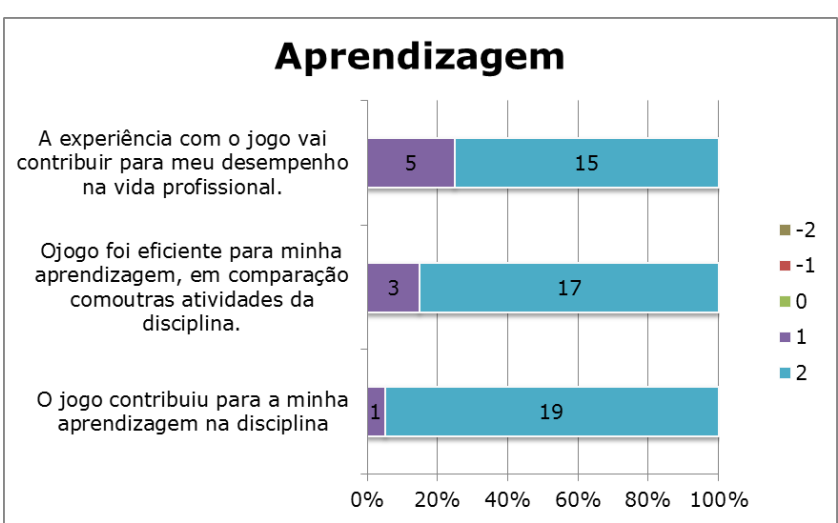

Figura 4: Avaliação geral da aprendizagem

Tabela 5: Avaliação da aprendizagem dos conteúdos no XP Enigma

\begin{tabular}{|c|c|c|c|}
\hline \multirow{2}{*}{$\begin{array}{l}\text { Nível cognitivo } \\
\text { Conteúdos }\end{array}$} & \multicolumn{3}{|c|}{ Aplicar } \\
\hline & Antes & Depois & Diferença \\
\hline Programação em Pares & 2,35 & 4,60 & $p<0.001$ \\
\hline Metáforas & 2,40 & 4,55 & $p<0.001$ \\
\hline Integração Contínua & 2,70 & 4,90 & $p<0.001$ \\
\hline Propriedade Coletiva & 2,70 & 4,40 & $p<0.001$ \\
\hline Design Simples & 2,60 & 4,80 & $p<0.001$ \\
\hline
\end{tabular}

Além das questões fechadas, os estudantes puderam descrever os principais pontos positivos e a melhorar do jogo. Dos vinte estudantes respondentes, os pontos positivos mais frequentemente mencionados foram relacionados à colaboração e interação (12), coletividade e trabalho em equipe (7), relação com a prática (7), reafirmação dos conceitos e aprendizagem (7). Os pontos de melhoria mais mencionados foram relacionados a colocar as pistas em locais diferentes para as diferentes equipes (5) e aumentar o número de equipes (3). Alguns comentários abertos e espontâneos, como do participante P2 ("Atividades em grupos e dinâmicas, isso fez com a teoria ficasse muito mais simples."), do participante P15 ("Muito dinâmico e divertido a forma de aprender, além de ajudar na socialização dos alunos.") e P17 ("Fez fixar os conhecimentos e ter argumentos para defender o método ágil"), enfatizam que a atividade, além de divertida e prazerosa, auxiliou na aprendizagem dos estudantes.

Além da avaliação pós-teste, foi realizada uma avaliação longitudinal (dois a quatro anos após a aplicação do jogo), a fim de identificar se os benefícios percebidos foram perenes, ou seja, persistiram ao longo do tempo.

\subsection{Avaliação Longitudinal}

Quanto à avaliação dos egressos, foi enviado um questionário para os 62 estudantes que tinham participado do jogo, dos quais 46 responderam, sendo 19 da turma 1 (2012), 15 da turma 2 (2013) e 12 da turma 3 (2014/2015), perfazendo $74,2 \%$ de participação.

Em questão aberta, foi perguntado para os estudantes "Quais as atividades/dinâmicas desenvolvidas em sala que você se recorda da disciplina?". Embora tenham sido realizadas diversas outras atividades, como dinâmicas, jogos e atividades vivenciais, dezenove estudantes $(41,3 \%)$ mencionaram espontaneamente o jogo XP Enigma.

De forma direcionada (mencionando o jogo), os estudantes avaliaram a motivação (quanto a atividade foi importante para manter o interesse na disciplina) e a aprendizagem (conhecimento e habilidades adquiridos). A Tabela 6 mostra esses resultados, indicando um alto índice de avaliações positivas, tanto da motivação quanto da aprendizagem, mesmo dois a quatro anos após a participação no jogo.

Tabela 6: Avaliação do XP Enigma pelos alunos egressos

\begin{tabular}{ccrrr}
\hline & \multicolumn{2}{c}{ Motivação } & \multicolumn{2}{c}{ Aprendizagem } \\
XP Enigma & Qtde & Percent. & Qtde & Percent. \\
\hline Ótimo & 22 & $47.8 \%$ & 19 & $41.3 \%$ \\
\hline
\end{tabular}




\begin{tabular}{c|rrrr}
\hline Bom & 13 & $28.3 \%$ & 16 & $34.8 \%$ \\
Regular & 8 & $17.4 \%$ & 6 & $13.0 \%$ \\
Péssimo & 0 & $0.0 \%$ & 1 & $2.2 \%$ \\
Não lembro & 3 & $6.5 \%$ & 4 & $8.7 \%$ \\
\hline
\end{tabular}

\section{Conclusões}

0 trabalho demonstrou um jogo para apoio ao ensino de metodologias ágeis, com ênfase em práticas do XP e SCRUM. 0 jogo, baseado na busca de uma mandala perdida, promove a realização, em equipe e iterações, de atividades que remetem a práticas ágeis.

0 artigo descreve o planejamento e execução do jogo em três turmas da disciplina de Métodos Ágeis, totalizando 62 estudantes. São descritos os resultados de uma avaliação pósteste com uma dessas turmas, totalizando 20 estudantes. Além disso, são descritos os resultados de uma pesquisa com 46 dos 62 estudantes, dois ou mais anos após a realização da atividade, para avaliar a percepção ao longo do tempo e a perenidade dos benefícios do jogo.

A atividade mostrou-se eficiente, indicando evolução do conhecimento prévio do assunto e mostrou-se uma atividade atrativa (96\% de aprovação), prendendo a atenção e provocando participação e engajamento.

Os únicos dois itens avaliados que tiveram avaliações negativas (somente duas) foram relacionados à evolução no jogo usando habilidades individuais. Uma possível razão para isso é que as atividades, em geral, são realizadas em duplas ou em equipe, tendo uma dependência do conhecimento e habilidade de outros membros da equipe e não exclusivamente de cada estudante.

Após dois anos ou mais da participação na disciplina e no jogo, os estudantes egressos confirmaram a avaliação positiva, tanto para a motivação que o jogo promoveu para dar continuidade nos estudos (76\% de avaliações positivas), quanto na aprendizagem promovida pelo jogo $(76 \%$ de avaliações positivas).

Esse trabalho vem a corroborar com diversas outras experiências da aplicação de estratégias ativas para a aprendizagem de métodos ágeis, confirmando a eficácia para aumentar a motivação e engajamento dos estudantes, assim como a aprendizagem. Por exemplo, numa análise de 43 estudos de caso que avaliaram jogos sérios em ES, Petri et al. [27] comprovam que estudantes perceberam uma contribuição positiva dos jogos na motivação, que a experiência de uso foi muito boa e que os jogos contribuíram positivamente para a aprendizagem. Além disso, o mesmo estudo mostrou não existir diferença significativa, em todos esses aspectos, entre jogos digitais e não digitais.

Weber [28] descreve um experimento com a aplicação e avaliação do "Extreme Construction" com 45 estudantes da disciplina de Gerenciamento de Projetos e Desenvolvimento Ágil. Da mesma forma que com o XP Enigma, foram realizadas avaliações com a auto percepção de aprendizagem antes e após o jogo. A Tabela 7 compara os resultados para as cinco práticas focadas no XP Enigma.
Tabela 7: Comparativo da evolução de aprendizagem

\begin{tabular}{ccccccc}
\hline & \multicolumn{3}{c}{$\begin{array}{c}\text { Extreme } \\
\text { Construction }\end{array}$} & \multicolumn{3}{c}{$\begin{array}{c}\text { XP } \\
\text { Enigma }\end{array}$} \\
\hline & Antes & Após & Var. & Antes & Após & Var. \\
\hline $\begin{array}{c}\text { Programação em } \\
\text { Pares }\end{array}$ & 4.22 & 4.56 & $8 \%$ & 2.35 & 4.60 & $96 \%$ \\
$\begin{array}{c}\text { Metáforas } \\
\text { Integração }\end{array}$ & 1.11 & 2.89 & $160 \%$ & 2.40 & 4.55 & $90 \%$ \\
$\begin{array}{c}\text { Contínua } \\
\text { Propriedade } \\
\text { Coletiva }\end{array}$ & 2.50 & 4.22 & $69 \%$ & 2.70 & 4.90 & $81 \%$ \\
Design Simples & 2.76 & 4.11 & $61 \%$ & 2.70 & 4.40 & $63 \%$ \\
\hline
\end{tabular}

Portanto, percebe-se que o XP Enigma teve valores da percepção de aprendizagem maiores para todas as práticas, mesmo sendo uma atividade de apenas 70 minutos, enquanto o Extreme Construction foi aplicado durante três meses. Embora para a prática "Metáforas" a evolução de aprendizagem do XP Enigma tenha sido menor, a percepção final da aprendizagem foi significantemente maior que o experimento utilizando Extreme Construction.

Assim como descrito por Souza et al. [21], abordagems relacionadas a jogos são úteis para apoiar a educação em Engenharia de Software, mas são geralmente descritas como recursos complementares, ao invés de único método de aprendizagem. Verificou-se que o jogo não deve ser a única estratégia de ensino utilizada, mas mostrou-se muito útil para a consolidação do conhecimento e, principalmente, para a aplicação da teoria numa atividade "mão na massa", mesmo não sendo no contexto de desenvolvimento de software propriamente dito. Schoeffel, Wazlawick e Ramos [29] também corroboram que o uso de múltiplas estratégias e metodologias ativas de ensino-aprendizagem têm um resultado efetivo no ensino de Engenharia de Software e Gerenciamento de Projetos.

Diante desses resultados e também da percepção de participação e engajamento dos estudantes durante a execução do jogo, tem-se evidências que o XP Enigma seja uma forma atrativa e eficiente para apoiar no ensino de métodos ágeis, principalmente de princípios e práticas do XP.

Como ameaças à validade do trabalho, tem-se: i) o número limitado de turmas e alunos avaliados; ii) a falta de variação do contexto, sendo realizadas na mesma universidade e curso; e iii) a auto avaliação dos estudantes pode gerar vieses nos dados de acordo com a veracidade das respostas. Como limitação principal, tem-se a dificuldade de replicação das mesmas atividades do jogo, devido a restrições do ambiente de aplicação, necessitando de adaptação de acordo com cada realidade.

Como trabalhos futuros, sugere-se a aplicação com outras turmas e instituições, comparando resultados, inclusive em nível de graduação. Sugere-se a adaptação da atividade para outras realidades e um estudo para tentar padronizar as atividades, para que possam ser realizadas em diferentes contextos e ambientes. Também sugere-se que seja avaliada a aprendizagem das demais práticas ágeis contempladas no jogo. 


\section{Referências}

[1] Standish Group, “Standish CHAOS Summary Report 2016," 2016.

[2] Digital.ai, "The 14th Annual State of Agile Report," VersionOne CollabNet, Alpharetta, GA, USA, 2020.

[3] VersionOne, “9TH Annual State of Agile Survey," 2015.

[4] I. Ibrahim, "Teaching Project Management for IT Students: Methods and Approach," em International Conference on Education and Management Technology, Singapore, 2011.

[5] U. Ojiako , M. Ashleigh e M. Chip, "Learning and teaching challenges in project management," International Journal of Project Management, vol. 29, p. 268-278, 2011.

[6] G. Bavota, A. D. Lucia e F. Fasano, "Teaching Software Engineering and Software Project Management: An Integrated and Practical Approach," em International Conference on Software Engineering (ICSE), Zurich, Switzerland, 2012.

[7] A. GOLDMAN, F. KON e P. J. S. e. Y. J. W. SILVA, "Being Extreme in the Classroom: Experiences Teaching XP," J. Braz. Comp. Soc., vol. 10, no 2, pp. 5-21, 2004.

[8] R. Hoda, J. Noble e S. Marshall, "Agile Project Management," em International Conference on Extreme Programming and Agile Processes in Software Engineering, Christchurch, New Zealand. , 2008.

[9] B. A. Hussein, "A Blended Learning Approach to Teaching Project Management: A Model for Active Participation and Involvement: Insights from Norway," Education Science, vol. 5, p. 104-125, 2015.

[10] T. D. Lynch, M. Herold, J. Bolinger, S. Deshpande, T. Bihari, J. Ramanathan e R. Ramnath, "An Agile Boot Camp: Using a LEGOß-Based Active Game to Ground Agile Development Principles.," em 41st ASEE/IEEE Frontiers in Education Conference, Rapid City, SD, 2011.

[11] A. Gkritsi, "Scrum Game: An Agile Software Management Game," 2011.

[12] C. G. v. Wangenheim, R. Savi e A. F. Borgatto, "SCRUMIA-An educational game for teaching SCRUM in computing courses," The Journal of Systems and Software, vol. 86, p. 2675- 2687, 2013.

[13] V. C. P. Peeters, "XP Planning Game," 2010. [Online]. Available: http://www.xp.be/xpgame.html. [Acesso em 2010].

[14] K. Beck, Extreme Programming Explained: embrace change, 2nd ed., Pearson Education, 2004.

[15] P. E. Battistella e C. G. v. Wangenheim, "Games for Teaching Computing in Higher Education - A Systematic Review," vol. 1, no 3, pp. 8-30, Março 2016.

[16] A. Bassi, "Scrum Sim - A Simulation Game to Learn the Scrum Agile Framework," 2016.

[17] A. Godoy, "Game-Scrum: An Approach to Agile Game Development," em SBC - Proceedings of SBGames 2010, Florianópolis - Brazil, 2010.

[18] W.-L. Lee, "SCRUM-X: An Interactive and Experiential Learning Platform for Teaching Scrum," em Proceedings of IMCIC - ICSIT 2016, 2016.

[19] A. Brito e J. Vieira, “'2TScrum': A Board Game to Teach Scrum," em Simpósio Brasileiro de Engenharia de Software (SBES'17), Fortaleza, Brazil, 2017.

[20] P. Rodrigues, M. Souza e E. Figueiredo, "Games and Gamification in Software Engineering: A Survey with Educators," 2018.

[21] M. Souza, L. Veado, R. T. Moreira, E. Figueiredo e H. Costa, "A systematic mapping study on game-related methods for software engineering education," A systematic mapping study on game-related methods for software, vol. 95, pp. 201-2018, 2018.

[22] J. Bergin e F. Grossman, "Extreme Construction: Making Agile Accessible," em Proceedings of AGILE 2006 Conference (AGILE'06), 2006.

[23] A. Przybyłek e M. Zakrzewski, "Adopting Collaborative Games into Agile Requirements Engineering," em Proceedings of the 13th International Conference on Evaluation of Novel Approaches to Software Engineering (ENASE 2018), 2019.

[24] D. Parsons, "Creating game-like activities in agile software engineering education," em 23rd Australasian Software Engineering Conference. ASWEC 2014, Sydney, Australia, 2014.

[25] R. Savi, C. G. von Wangenheim e A. F. Borgatto, "A Model for the Evaluation of Educational Games for Teaching Software Engineering," em 25th
Brazilian Symposium on Software Engineering, SBES 2011, Sao Paulo, Brazil,, 2011.

[26] M. Tavakol e R. Dennick, "Making sense of Cronbach's alpha," International Journal of Medical Education, vol. 2011, no 2, pp. 53-55, 2011.

[27] G. Petri, C. G. v. Wangenheim e A. F. Borgatto, "Quality of Games for Teaching Software Engineering: An Analysis of Empirical Evidences of Digital and Non-digital Games," em 2017 IEEE/ACM 39th International Conference on Software Engineering: Software Engineering Education and Training Track, 2017.

[28] B. Weber, "Extreme Construction Game to Improve the Teaching of Extreme Programming Practices," Austria.

[29] P. Schoeffel, R. S. Wazlawick e V. F. C. Ramos, "Using Multiple Active Teaching-Learning Approaches in Software Project Management: A longitudinal analysis of students' motivation and learning," em 2018 IEEE Frontiers in Education Conference (FIE), San Jose, CA, USA, 2018.

[30] J. M. Fernandes e S. M. Sousa, "PlayScrum - A Card Game to Learn the Scrum Agile Method," em 2010 Second International Conference on Games and Virtual Worlds for Serious Applications, 2010.

[31] K. Greaves e S. Laing, "Collaboration Games from the Growing Agile Toolbox," Growing Agile, 2013. 


\section{Apêndice I - Dicas utilizadas no XP Enigma}

\begin{tabular}{|c|c|c|}
\hline Dica & Descrição & Resposta \\
\hline 1 & $\begin{array}{l}\text { ARTES: Você precisará se localizar no campus/sala para algumas atividades. Faça uma planta do campus numa } \\
\text { folha de papel }\end{array}$ & Desenho \\
\hline 2 & MATEMÁTICA e GEOMETRIA: Figura geométrica em 3 dimensões com a uma base e 3 lados iguais & Triângulo \\
\hline 3 & $\begin{array}{l}\text { GERAIS: Nome dado a mulher com cabelo ruivo (popular) + Nome da filha adolescente da família que } \\
\text { protagoniza o seriado "Os Simpsons" }\end{array}$ & Mona+Lisa \\
\hline 4 & INTEGRAÇÃO: Primeira letra do nome próprio do local representado pelas dicas 2 e 3 & $\begin{array}{l}\text { L - Museu } \\
\text { do Luvre }\end{array}$ \\
\hline 5 & $\begin{array}{l}\text { LÓGICA: Letra correspondente à soma dos números da próxima sequência do enigma abaixo, diminuindo } 1 . \\
21 \\
1211 \\
111221 \\
312211 \\
13112211 \\
? ? ? ? ? ? ? ? ? ? ?\end{array}$ & $\begin{array}{l}1113212221 \\
(\text { Soma }=20, \\
\text { Letra }=\mathrm{S})\end{array}$ \\
\hline 6 & $\begin{array}{l}\text { LÓGICA: Informação representada pelos } 2^{\circ} \text { e } 3^{\circ} \text { primeiros símbolos da seguinte charada } \\
\text { LÓGICA: Informação representada pelos } 2^{\circ} \text { e } 4^{\circ} \text { símbolos da seguinte charada }\end{array}$ & $\begin{array}{l}12 \text { e } 13 \\
\text { (números } \\
\text { espelhados) }\end{array}$ \\
\hline 7 & GERAIS: Letra inicial do país campeão da Copa do Mundo de Futebol de 1990 & $\begin{array}{l}\text { (A } \\
\text { Alemanha) }\end{array}$ \\
\hline 8 & ARTES: Identifique o número das salas no desenho da planta do campus / detalhes da sala & Desenho \\
\hline 9 & $\begin{array}{l}\text { INTEGRAÇÃO: Dica } 5+\text { Dica } 7 \text { + Dica } 4 \text { + Dica } 7 \text { + Dica } 6 \text { representa o local, o acesso a esse local está } \\
\text { representado na dica } 10\end{array}$ & $\begin{array}{l}\text { SALA } 12 \text { ou } \\
\text { SALA } 13\end{array}$ \\
\hline 10 & GERAIS: sou personagem de um filme lançado na década de 70 e sou conhecido pelo número 53 & $\begin{array}{l}\text { Herbie } \quad- \\
\text { fusca }\end{array}$ \\
\hline 11 & ARTES: identifique os pontos cardeais no desenho da planta do campus & Desenho \\
\hline 12 & $\begin{array}{l}\text { GERAIS: “Abertura para entrar ou sair" + "Peça de roupa ou Pequeno tubo com roscas internas e que serve } \\
\text { como conexão entre canos } \\
\text { GERAL: “Abertura para entrar ou sair" + "Diz-se da pessoa chata, inconveniente, sem graça ou espécie de } \\
\text { caixa com alças }\end{array}$ & $\begin{array}{l}\text { Porta-luvas } \\
\text { Porta-malas }\end{array}$ \\
\hline 13 & $\begin{array}{l}\text { INTEGRAÇÃO: Dica } 10+\text { Dica 12. Pegue somente o acesso correspondente ao local da sua equipe, sob pena } \\
\text { de perder o jogo }\end{array}$ & $\begin{array}{l}\text { Porta-malas } \\
\text { ou porta- } \\
\text { luvas } \\
\text { Fusca }\end{array}$ \\
\hline 14 & $\begin{array}{l}\text { ENIGMA: Não penso nem reflito, mas conhecimento eu transmito, como se fosse uma lua irradiando sua } \\
\text { claridade numa noite escura. }\end{array}$ & $\begin{array}{l}\text { Projetor } \\
\text { multimídia }\end{array}$ \\
\hline 15 & $\begin{array}{l}\text { GEOGRAFIA: estou para o campus assim como o Ushuaia está para a América....Sou pequena e tenho } 2 \\
\text { compartimentos }\end{array}$ & $\begin{array}{l}\text { Lixeira } \\
\text { posicionada } \\
\text { no sul do } \\
\text { campus }\end{array}$ \\
\hline
\end{tabular}

